\title{
Laser Doppler Flowmetry in the Mierofymphodynamics Study
}

DOI: $10.17691 / \mathrm{stm} 201911.2 .13$

Received March 20, 2018

\section{P.V. Vasilev, PhD Student:}

N.B. Margaryants, PhD, Senior Teacher, Faculty of Applied Optics;

N.P. Erofeey, MD, DSC, Professor, Department of Physiology'

1 Saint Petersburg State University, 7/9 Universitetskaya Naberezhnaya, Saint Petersburg, 199034, Russia;

2 Saint Petersburg National Research University of Information Technologies, Mechanics and Optics, 49 Kronverksky Avenue, Saint Petersburg, 197101, Russia

Microcirculatory bed, as part of the whole human vascular system, is the link between blood, lymph, and interstitial space. Laser Doppler flowmetry (LDF) is traditionally used to study blood microcirculation.

The aim of the study was to identify the wavelength ranges in which the differences in the reflection coefficient of vessels with different degrees of blood and lymph filling are maximal. The nature of the differences in the reflection coefficient may allow the estimation of the contribution of the blood and lymphatic components to the total reflected signal.

Materials and Methods. The reflection coefficient on isolated blood and lymphatic vessels in the wavelength ranges commonly used in existing diagnostic systems has been investigated, the amplitude-frequency parameters of LDF signals characterizing the functional state of the blood and lymph flows have also been analyzed.

The experiments were carried out on laboratory male Wistar rats. Static spectral characteristics of blood and lymph have been studied on isolated vessels obtained in the acute experiment using HR4000 spectrometer (Ocean Optics, USA). The portal vein and thoracic lymphatic duct of the living anesthetized rat have been selected as an object for studying LDF characteristics of the blood and lymph flow in vivo. Biopac LDF 100C diagnostic system (Biopac Instruments, USA) with a probe wavelength of $830 \pm 10 \mathrm{~nm}$ was used for measurements.

Results. After the evacuation of blood or lymph in the isolated vessels, significant changes $(p=0.0059)$ in the reflection coefficient in certain wave ranges (700-860 nm for lymphatic and 410-560 nm for blood vessels) have been registered that, in our opinion, allowed us to evaluate the dynamics of filling the probed object with blood or lymph, respectively. During heart contraction, a LDF signal with phase oscillations has been recorded on the thoracic lymphatic duct persisting after cardiac arrest. Its amplitude-frequency spectrum contained the predominant slow-wave harmonics increasing in cardioplegia.

Conclusion. The results obtained demonstrate the possibility of the LDF method to record the signal characterizing the change in tissue perfusion due to the lymphatic flow. The spectral reflective properties of the isolated vessels are characterized by a multidirectional change in the reflection coefficient with a decrease in blood and lymph concentration in the studied tissue volume that should be taken into account when choosing a radiation source for LDF studies and developing new techniques of functional tests.

Key words: laser Doppler flowmetry; blood microcirculation; lymph microcirculation; probing radiation; spectral analysis.

\section{Introduction}

Microcirculatory bed, as part of the whole human vascular system, is the link between the compartments of the internal body environment, i.e. blood, lymph, and interstitial space, and is vulnerable structurally and functionally to external and/or internal negative impacts.

Laser Doppler flowmetry (LDF) has been traditionally (since the adoption to clinical practice) used to investigate microhemocirculation [1-5]. A specific feature of this method consists in the fact that a blood flow in a separate vessel is not the substrate to be measured but rather a general microcirculation picture of perfusion in the biotissue region being probed representing a superposition of multidirectional particle movements in a large number of microvessels and changes in the concentration of these particles in the examined region
$[2,3]$. Numerous researchers working in this direction have developed various methods of microcirculation analysis using LDF, they have established the rhythms of bloodstream oscillations in the microcirculatory bed and their diagnostic value [1-3, 6-10]. At the same time, works devoted to LDF application for microlymphodynamics assessment are at present rather scarce [11-15].

The specificity of our work is to study separately optical characteristics of vascular segments and to analyze the spectral composition of the LDF signal recorded on isolated blood and lymphatic vessels.

The aim of the study was to identify and justify the wavelength ranges of the probe radiation in order to assess hemolymphodynamics by the LDF method on the basis of simultaneous comparative experimental investigation of the reflection coefficient and amplitude- 
frequency characteristics of the LDF signal on the isolated segments of the blood and lymphatic systems.

The following tasks were to be solved in the course of the investigation:

to identify the wavelength ranges of the probe radiation with maximal differences of the reflected signal depending on the filling of the blood or lymphatic vessel and optimal for blood and lymph flow assessment;

to perform comparative analysis of the amplitudefrequency values of the LDF signal on the isolated blood and lymphatic vessels in vivo.

\section{Materials and Methods}

The experiments were carried out by the Department of Physiology of the Saint Petersburg State University on male Wistar rats aged 5-6 months weighing 180-200 g.

The experiments were conducted in compliance with the European Convention for the Protection of Vertebrate Animals used for Experimental and Other Scientific Purposes (Strasbourg, 2006).

Investigations of spectral characteristics of blood and lymph in isolated vessels. Static spectral characteristics of blood and lymph were studied on the isolated vessels obtained in the acute experiment from common laboratory Wistar rats (males aged 5-6 months, $n=5)$. Segments of the thoracic lymphatic duct and inferior vena cava containing lymph and blood, respectively, were extracted from the etheranesthetized rat.

These large vessels were chosen for the experiment because the device capabilities make it impossible to focus the signal only on lymphatic or blood capillaries. The size of the large vessel corresponds to the probing zone of the LDF system making it possible to register separate signals from the blood or lymphatic vessel.

The extracted specimens were placed on the special black substrate over which optical fibers from the radiation source and photodetector were rigidly fixed with a metal clamp (Figure 1). The specimen was constantly irrigated with $0.9 \%$ solution of sodium chloride to prevent drying.

A tungsten halogen lamp HL-2000 (Ocean Optics, USA) served as a radiation source. A fiber optic receiver was connected to the HR4000 spectrometer (Ocean Optics, USA). The investigations were conducted in the spectral range of $0.4-0.86 \mu \mathrm{m}$.

First, the reflection spectrum of the vessel filled with a biological medium (blood or lymph) was recorded. Then one of the ligatures was cut off, blood and lymph flowed out of the vessel, and the reflection spectrum was registered again.

Investigation of the LDF signal from the blood and lymph flow in vivo. In this part of the investigation, 5 male Wistar rats were used. Here, the portal vein and thoracic lymphatic duct were chosen as the objects for examining LDF characteristics of the blood and lymph flow in vivo. The Biopac LDF 100C diagnostic system

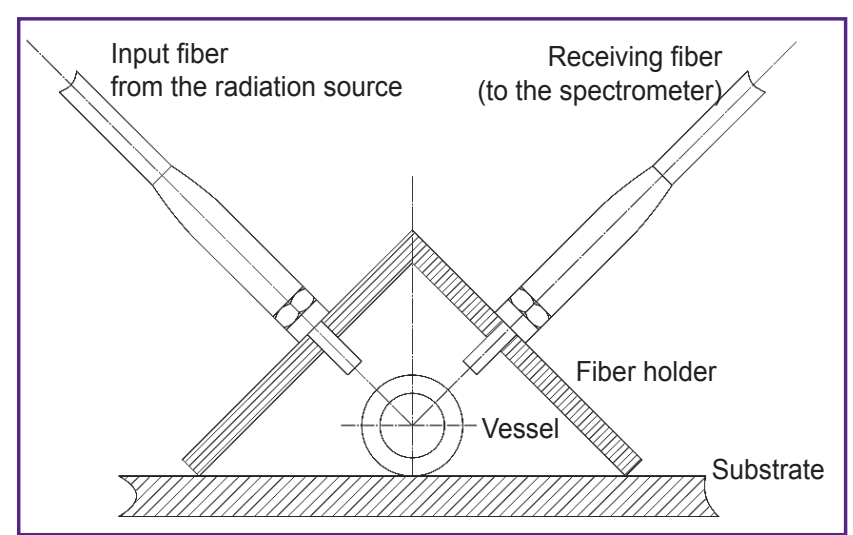

Figure 1. The system for investigation of an isolated vessel

(Biopac Instruments, USA) with $830 \pm 10 \mathrm{~nm}$ of the probe wavelength was used for measurements.

The abdominal and thoracic cavities were opened in a diethyl ether-narcotized rat, the portal vein and thoracic lymphatic duct were isolated with the help of traction sutures. The LDF signal was recorded for 2 min alternately on both vessels using TSD 144 needle sensor.

Then, cardiac arrest was achieved by continuing the exposure of the rat to the diethyl ether. When blood circulation was stopped the repeated 2-minute signal registration was performed over the thoracic lymphatic duct and portal vein, the signal from the intestinal lymphatic trunk was also additionally recorded.

To process the obtained LDF signals, mean values of perfusion and its standard deviation (flux) were calculated. Then the amplitude-frequency spectra were built using AcqKnowledge 3.8.2. program.

Data were statistically processed by means of the GraphPad Prism 6.0 software package designed for statistical analysis. To compare the intergroup values, unpaired non-parametric Mann-Whitney test was used.

\section{Results and Discussion}

Experiments on isolated vessels. The reflection spectra of the isolated thoracic duct and inferior vena cava are shown in Figure 2. One curve corresponds to the reflection spectrum of the filled vessel. After blood or lymph evacuation, statistically significant $(p=0.0059)$ changes of the reflection coefficient were found in certain wavelength ranges $(700-860$ and $410-560 \mathrm{~nm})$ which is demonstrated by another curve.

In our opinion, the reflection coefficient difference in the mentioned ranges makes it possible to assess the dynamics of filling the probed objects with blood or lymph, respectively.

In all experiments with the blood vessels, differences between the reflection coefficients were found in two spectral ranges: $410-560$ and $700-860 \mathrm{~nm}$, whereas for lymphatic vessels only in the $700-860 \mathrm{~nm}$ range. 


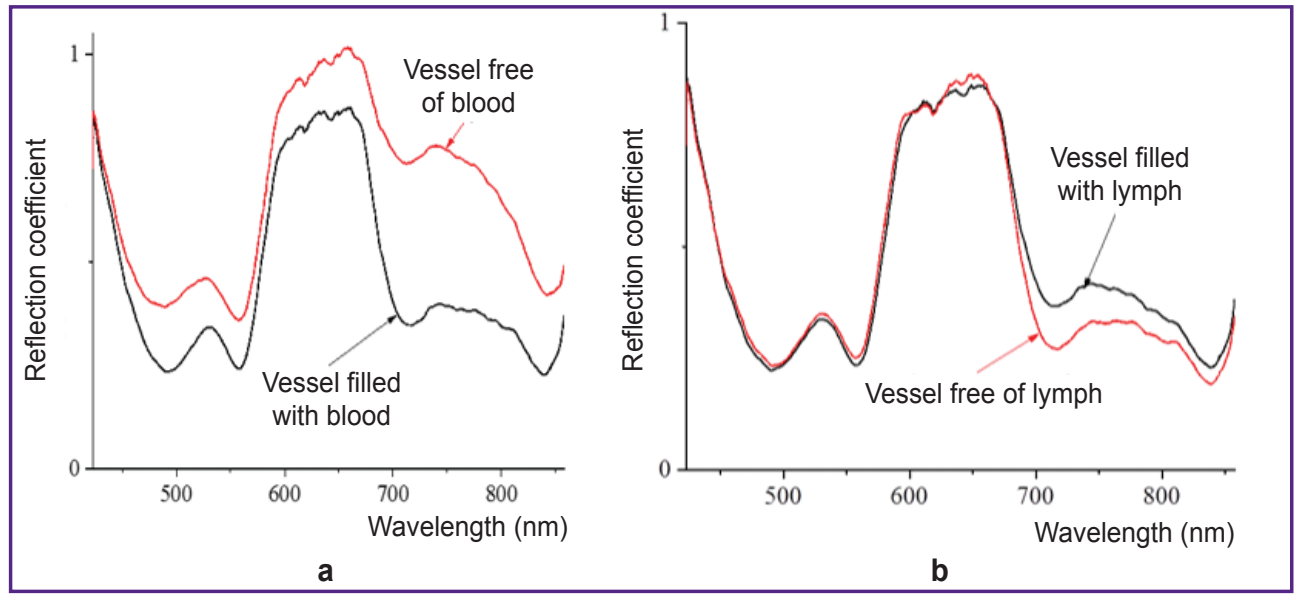

Figure 2. Reflection spectra of the blood and lymphatic vessels:

(a) inferior vena cava; (b) thoracic lymphatic duct

There are plenty of researches in which LDF is applied for microhemocirculation analysis. The diagnostic systems used in those cases (Biopac; Biopac Systems, USA; LAKK; Lazma, Russia, and others) have a probe radiation wavelength within the range of 700$860 \mathrm{~nm}(830 \mathrm{~nm}$ for Biopac, $780 \mathrm{~nm}$ for serial LAKK, and so on).

The detected differences between the reflection coefficients in the $700-860 \mathrm{~nm}$ range bear a multidirectional character for blood and lymphatic vessels. In the blood vessels, it manifests itself with the increase of the vessel reflection coefficient and the decrease of blood filling. In the lymphatic vessels, the decrease of the lymph filling, on the contrary, results in the decrease of the reflection coefficient. The value of this difference characterizes the contribution of the blood and lymphatic components of microcirculatory bed perfusion.

LDF investigations in vivo. LDF signals shown in Figure 3 were recorded on the thoracic lymphatic duct when the heart was contacting and arrested.

Phase oscillations could be noted in these signals. An average level of perfusion index in case of the contracting heart was 290 blood perfusion units (BPU) at the flux value of 20 BPU. In case of cardioplegia and persistence of phase oscillations, the value of perfusion index reduced to $124 \mathrm{BPU}(p=0.023)$ at the flux value of $11 \mathrm{BPU}(p>0.05)$. This phenomenon is likely to occur due to the exclusion of

Figure 4. LDF signal on the portal vein:

(a) contracting heart; (b) arrested heart the systemic factors of lymphodynamics (the suction action of the thorax and heart) though, on the whole, the level of the modulating actions (the contractile activity of lymphangions) remains on the same level.

The signals registered on the portal vein are shown in Figure 4. When recording the LDF signal (in case of the contracting heart) the curve has a distinct wave-like character with the frequency corresponding to the heart

rate. The mean value of the perfusion

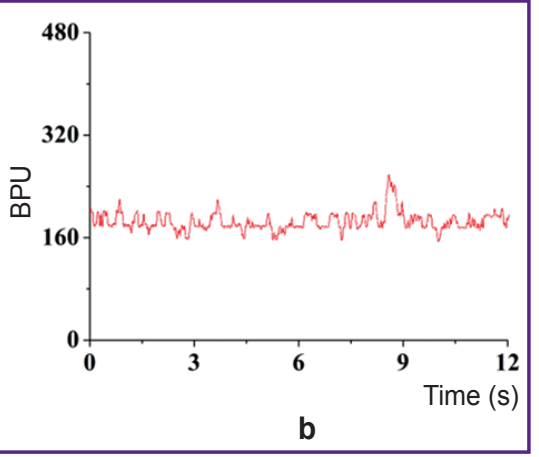


index, in this case, is $185 \mathrm{BPU}$ at the flux value of 69 BPU. The curve becomes chaotic, variations of the perfusion index are not stable when the signal was registered on the portal vein under the condition of the arrested heart. The mean value of the perfusion index is significantly lower (94 BPU $(p=0.027)$ ), the flux value also reduces significantly $-13 \mathrm{BPU}$ $(p=0.036)$.

Thus, a large value of flux is primarily characteristic of the signal from the blood vessel for case of the contracting heart. Since the flux characterizes the dispersion of perfusion index variations under the impact of diverse factors of bloodstream modulation, it may be concluded that a high flux in the signal from the blood vessel is caused by the predominant importance of the heart work with its propulsively sucking action. When the heart is arrested, this factor is excluded leading to the reduction of the flux and perfusion index values.

The assessment of the spectral characteristics of the signal from the portal vein registered when the heart was contracting shows that a slow-wave harmonic in the spectrum (in the range of $0.05-0.2 \mathrm{~Hz}$ ) is not revealed. The nearest to the $\mathrm{Y}$-axis harmonic is

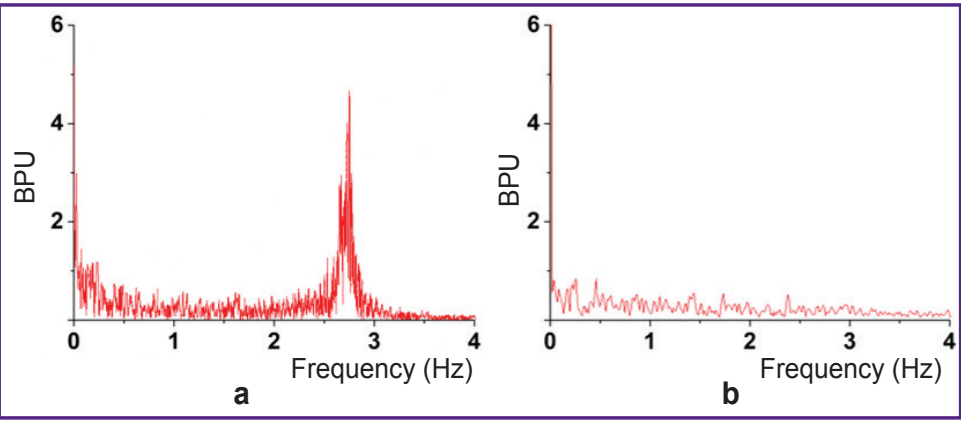

Figure 5. Amplitude-frequency spectrum of the venous signal:

(a) contracting heart; (b) arrested heart

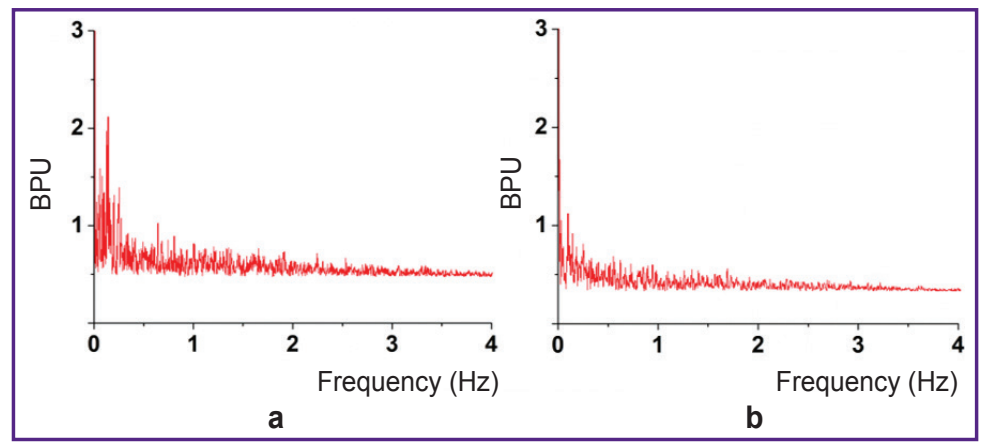

Figure 6. Amplitude-frequency spectrum of the thoracic duct signal: (a) contracting heart; (b) arrested heart noted beginning with the frequency of $0.3 \mathrm{~Hz}$. A pulse harmonic at 2.53-2.59 Hz frequency dominates in the spectrum, which corresponds to the heart rate of 150-155 per minute. When the heart is not working, no evident harmonics are noted in the spectrum of the venous signal (Figure 5).

In the spectrum of the lymphatic signal in case of the contracting heart, a prominent slow-wave harmonic is present, its average amplitude is $0.68 \pm 0.12 \mathrm{BPU}$ (at the maximum amplitude of $2.09 \mathrm{BPU}$ ). When the heart is arrested, the average amplitude of the slowwave region $(0.05-0.2 \mathrm{~Hz})$ in the amplitude-frequency spectrum of the signal registered on the thoracic duct is $0.36 \pm 0.08 \mathrm{BPU}$ (at the maximum amplitude of $1.02 \mathrm{BPU}$ ) (Figure 6).

Thus, the diagnostic LDF system with the probe radiation wavelength of $830 \pm 10 \mathrm{~nm}$ shows the capability of registering both lymph and blood flow. The lymph flow is registered under both conditions: contracting and arrested heart. The lymph movement under the action of lymphangions forms the amplitude harmonic in the wavelength range of $0.05-0.20 \mathrm{~Hz}$.

Notable, that this region in the majority of the investigations is designated as a range characterizing functioning of the local mechanism of bloodstream modulation (myogenic, neurogenic, and endothelial) $[1,8,13,15]$. Taking our data into consideration, it may be concluded that during LDF investigations with the sensor located on the patient's skin, the resultant signal represents a superposition of the signals from both blood

and lymph components. Therefore, it is necessary to reassess the existing algorithms of interpreting the lowwave harmonic amplitude and to develop mathematical methods of distinguishing the lymphatic and blood components of the LDF signal.

\section{Conclusion}

The results of the in vivo investigations of the spectral constituents of the microcirculatory bed oscillations using the LDF method for measuring the velocity of blood and lymph movement and the ex vivo study of the dynamics of reflection spectra of the blood and lymphatic vessels when measuring blood and lymph volume demonstrate that the LDF method allows the registration of the signal characterizing the changes in tissue perfusion due to the lymph filling. The spectral reflective properties of the isolated vessels are, in their turn, characterized by a multidirectional change in the reflection coefficient with a decrease in blood and lymph concentration in the studied tissue volume that should be taken into account when choosing a radiation source for LDF studies and developing new techniques of functional tests.

Study funding. The work was not funded by any source.

Conflicts of interest. There are no conflicts of interest related to this study. 


\section{References}

1. Bernjak A., Stefanovska A. Pulse transit times to the capillary bed evaluated by laser Doppler flowmetry. Physiol Meas 2009; 30(3): 245-260, https://doi.org/10.1088/0967$3334 / 30 / 3 / 002$.

2. Orlov L.V. Laser Doppler flowmetry in medical practice. Kazanskij medicinskij zurnal 2002; 83(3): 217-218.

3. Kozlov V.I., Azizov G.A., Gurova O.A., Litvin F.B. Lazernaya dopplerovskaya floumetriya $v$ otsenke sostoyaniya $i$ rasstroystv mikrotsirkulyatsii krovi [Laser Doppler flowmetry in assessing the condition and disorders of blood microcirculation]. Moscow; 2012.

4. Krupatkin A.I., Sidorov V.V. Funktsional'naya diagnostika sostoyaniya mikrotsirkulyatorno-tkanevykh sistem: kolebaniya, informatsiya, nelineynost' [Functional diagnosis of microcirculatory-tissue systems: oscillations, information, nonlinearity]. Moscow: URSS; 2016.

5. Fedorovich A.A. Microcirculation of the human skin as an object of research. Regionarnoe krovoobrashchenie i mikrotsirkulyatsiya 2017; 16(4): 11-26, https://doi. org/10.24884/1682-6655-2017-16-4-11-26.

6. Abramovich S.G., Mashanskaya A.V. Laser Doppler flowmetry in estimation of microcirculation in healthy people and patients with arterial hypertension. Sibirskij medicinskij zurnal 2010; 92(1): 57-59.

7. Gerasymchuk P.A., Kisil P.V., Vlasenko V.G., Pavlyshyn A.V. Endothelial dysfunction indicators in patients with diabetic foot syndrome. Vestnik Rossiyskoy akademii meditsinskikh nauk 2014; 69(5-6): 107-110.

8. Tankanag A.V., Chemeris N.K. Adaptive wavelet analysis of oscillations in the human peripheral blood flow.
Biophysics 2009; 54(3): 375-380, https://doi.org/10.1134/ s0006350909030221.

9. Petrov S.V., Kozlov V.I., Azizov G.A. Laser Doppler flowmetry in the complex examination of patients with chronic venous insufficiency. Lazernaya meditsina 2008; 12(2): 36-41.

10. Fredriksson I., Larsson M., Nystrom F.H., Lanne T., Ostgren C.J., Stromberg T. Reduced arteriovenous shunting capacity after local heating and redistribution of baseline skin blood flow in type 2 diabetes assessed with velocity-resolved quantitative laser Doppler flowmetry. Diabetes 2010; 59(7): 1578-1584, https://doi.org/10.2337/db10-0080.

11. Ladozhskaya-Gapeenko E.E., Bubnova N.A., Erofeev N.P., Katsev V.M., Kanina L.Ya. Diagnosis of low extremity lymphedema using laser Doppler flowmetry. Regionarnoe krovoobrashchenie i mikrotsirkulyatsiya 2011; 10(1): 20-28.

12. Krupatkin A.I. Oscillatory processes in lymph microcirculation in the human skin. Hum Physiol 2014; 40(1): 52-57, https://doi.org/10.1134/s0362119713040087.

13. Krupatkin A.I., Sidorov V.V. New perspectives of noninvasive estimation of microlymphocirculation with laser technologies. Vestnik limfologii 2014; 4: 21-28.

14. Krupatkin A.I., Sidorov V.V. The problem of adaptation and oscillatory processes in the microvascular bed. Hum Physiol 2016; 42(4): 408-415, https://doi.org/10.1134/ s0362119716040095.

15. Dremin V.V., Kozlov I.O., Zherebtsov E.A., Makovik I.N., Dunaev A.V., Sidorov V.V., Krupatkin A.I. The capabilities of laser Doppler flowmetry in assessment of lymph and blood microcirculation. Regionarnoe krovoobrashchenie i mikrotsirkulyatsiya 2017; 16(4): 42-49, https://doi. org/10.24884/1682-6655-2017-16-4-42-49. 